\title{
Tumor-associated collagen signatures: pushing tumor boundaries
}

\author{
Elizabeth A. Brett, Matthias A. Sauter, Hans-Günther Machens and Dominik Duscher ${ }^{*}$
}

\begin{abstract}
In 2006, a new model of invasive breast tumor emerged and, since 2011, is gaining recognition and research momentum. "Tumor-associated collagen signatures" describe 3 distinct layers of collagen which radiate outward in shells from the main body of the tumor. The outermost layer (TACS3) features branches of collagen radiating away from the tumor, $90^{\circ}$ perpendicular to the tumor surface. TACS3 increases tumor span and correlates directly with metastasis, though presently difficult to detect in breast tissue. TACS is an emerging model but has been validated by multiple labs in vitro and in vivo, specifically for breast cancer prognostics. Newly recognized and accepted tumor borders will impact both R0 resections and downstream surgical reconstruction. This review aims to comprehensively introduce and connect the ranging literature on linearized collagen of invasive tumor borders. Using PubMed keyword searches containing "aligned," "linear," "oriented," and "organized," we have gathered the studies on TACS, integrated the concept into the clinic, and projected future platforms.
\end{abstract}

Keywords: Tumor-associated collagen signatures, TACS, Breast cancer, MDA-MB-231, Oncogenic, Metastasis, Collagen, Tumor

\section{Introduction}

Understanding invasive breast tumor maturation has been the focus of breast cancer research for decades. Since 2006, there has been growing evidence of a previously unrecognized tumor feature which correlates with decreased patient survival rates. Tumor-associated collagen signatures (TACS) is a system which classifies three different collagen shells of the invasive breast tumor. Importantly, the outermost layer (TACS3) is defined by linear branches of collagen growing perpendicularly away from invasive breast tumors [1]. Within breast cancer literature, words like "aligned," "oriented," "organized," and "linear" can be found referring to a specific extracellular matrix $(E C M)$ pattern of the invasive breast tumors [2-4]. Clinically, there is a well-established link between the collagen architecture of the primary tumor and prognosis. In both canine and human mammary carcinomas

\footnotetext{
* Correspondence: dominik.duscher@tum.de

Department of Plastic and Hand Surgery, Technical University Munich, Ismaninger Strasse 22, 81675 Munich, Germany
}

(ductal and lobular), tumor borders with perpendicular protrusions of collagen correlated with metastasis, and TACS3-positive patients had statistically lower diseasefree survival. Lymphatic and vascular invasion and increased syndecan-1 presence were also correlated with TACS3 in biopsy samples [5, 6]. While TACS is not a perfect diagnostic tool for other tumor features like hormone receptor status, it is explained in the later sections of this review that TACS3 studies largely feature triplenegative breast cancer cells, thereby removing ER, PR, and HER2 from the diagnostic/therapeutic options.

The aims of this literature review are to collect existing studies on TACS, assess what TACS means for the clinic, and consider future directions.

\section{Tumor-associated collagen signatures}

In 2006, tumor collagen heterogeneity was described in the breast tumor using a tri-part system called "tumorassociated collagen signatures" or TACS. The classification breaks the tumor collagen into three zones, each of

C The Author(s). 2020 Open Access This article is licensed under a Creative Commons Attribution 4.0 International License, which permits use, sharing, adaptation, distribution and reproduction in any medium or format, as long as you give appropriate credit to the original author(s) and the source, provide a link to the Creative Commons licence, and indicate if changes were made. The images or other third party material in this article are included in the article's Creative Commons licence, unless indicated otherwise in a credit line to the material. If material is not included in the article's Creative Commons licence and your intended use is not permitted by statutory regulation or exceeds the permitted use, you will need to obtain permission directly from the copyright holder. To view a copy of this licence, visit http://creativecommons.org/licenses/by/4.0/ The Creative Commons Public Domain Dedication waiver (http://creativecommons.org/publicdomain/zero/1.0/) applies to the data made available in this article, unless otherwise stated in a credit line to the data. 
them physically distinct from one another [1]. TACS1 describes the inside layer of a densely packed collagen. TACS2 is a series of spheroidal shells around the TACS1 layer. TACS3 is defined by linear outgrowths of collagen leading from the tumor into breast parenchyma (Fig. 1). Many research papers which examine TACS are corollary in nature, with a large focus on describing the presence and prognostic value of TACS3. Our group recently published the linear formation of collagen in vitro by a breast cancer cell co-culture, which marks the first TACS publication to have a laboratory intervention experiment pertaining to linearized ECM of breast cancer [7]. TACS is largely described in breast tumors; however, orderly collagen formation is also seen in malignant ovarian tumors, with one study showing "increased normality" of collagen deposition [8]. While "collagen alterations" are seen as aligned waves of ECM in advanced ovarian tumors [9], no classification such as TACS has been adopted for this tumor or any other.

\section{TACS detection}

Second-harmonic generation (SHG) microscopy is a nonlinear microscopy technique which has been developed to enhance fibrosis research. The microscope structure is similar to that of an inverted light microscope and functions to analyze excised tissue en bloc. Briefly, a highenergy laser penetrates the sample, and when it meets the collagenous structures of specific orientation, the incident ray emitting from the sample is doubled in frequency. The ray is detected, interpreted through a series of formulae, and given a numerical value corresponding to isotropy/anisotropy [10]. Specifically, SHG can provide in-depth detail on fibrillar collagen and is useful in fibrosis, tumor, and connective tissue disorder research and diagnostics [11]. Limitations of the technique are the penetration depth, which is dependent on the scattering ability of the tissue (usually on the order of hundreds of micrometers). Similarly, it does not provide contrast for cells and is only highly specific for fibrillar collagen. While a low-cost version of a SHG microscope has been created, it still ranges around USD 40,000 [12]. Alternatives exist, for instance, spatial light interference microscopy (SLIM) has demonstrated better TACS3 detection along with better discernment of the tumor border, due to SLIM ability to measure linear and also non-linear collagen $[3,13]$. The value of SHG may be in the binary confirmation of the presence/ absence of linear collagen, as seen in past research which visualized the TACS3 collagen pattern in intact, unfixed human mammary gland samples [10]. For instance, SHG very successfully images the uterine wall tissue which is known for its linearity [14]. TACS is detectable using strategic histological sections made transversely at the tumor border. Recently, TACS3 at the tumor border of invasive breast tumors have been easily identified using classic hematoxylin and eosin staining [7]. However, this stain is only specific for collagen, not collagen isoforms. The same study used a parallel in vitro collagen deposition study and mass spectrometry to make an educated guess at which the isoform of collagen is highest in the TACS3 signatures. The proteome analysis of in vitro collagen showed collagen VI, which was seen using immunohistochemistry to be highly present in TACS3 of human tumor biopsy samples [7]. This represents the first reported biochemical target of TACS3. While more corroborative research needs to be published, visualizing collagen VI

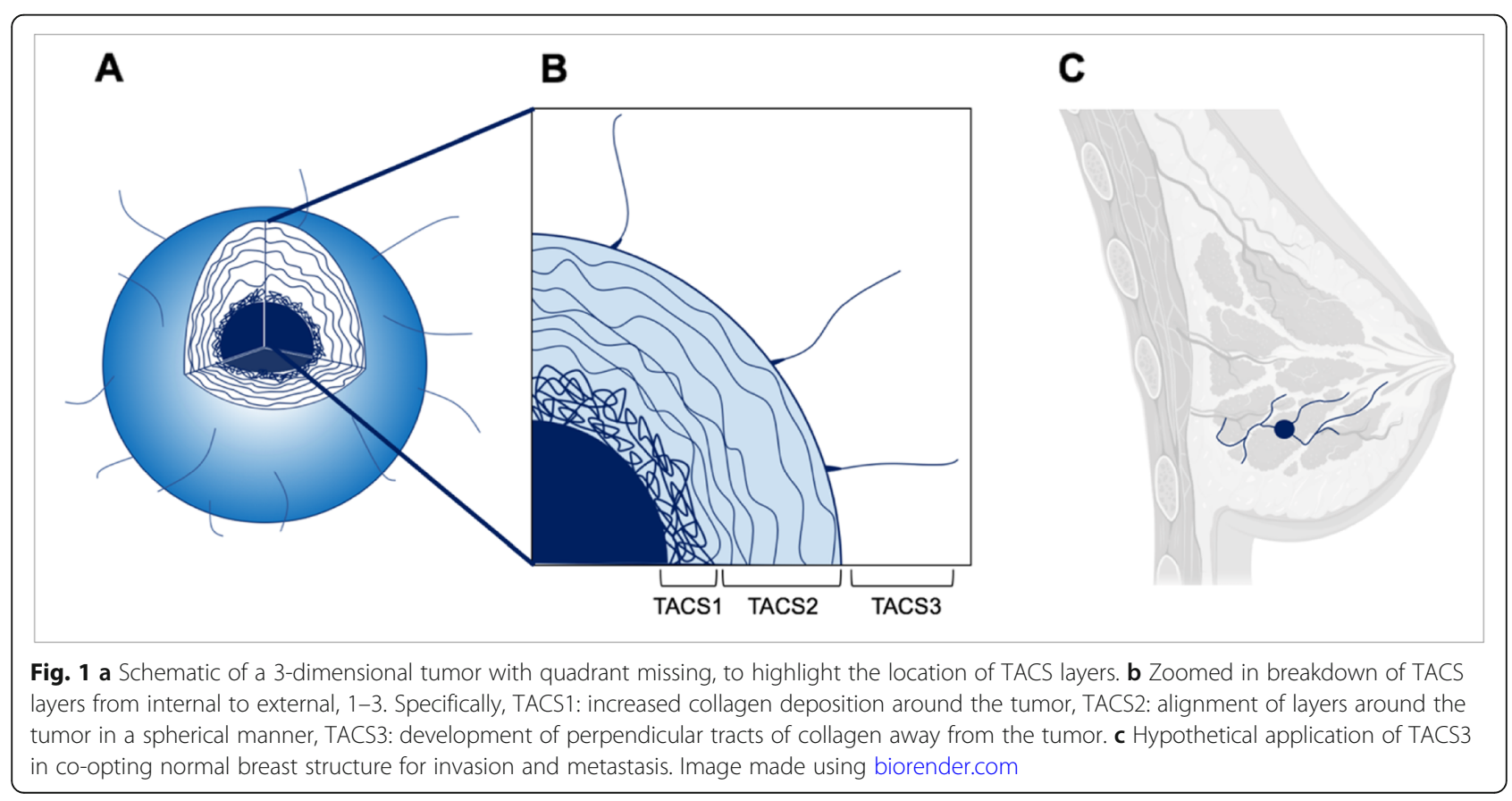


could represent a logical workaround to easy TACS3 detection, without having to source SHG.

\section{TACS in vitro}

Many experiments in vitro assay show the preferential formation of lines/striated structures by non-cancer cells and triple-negative breast cancer cells. For instance, MDA-MB-231 cells cultured in two points opposite of each other over 10 days showed a reorganization of the collagen gel in between, from random formation to a linear pattern [15].. Linear manipulation of collagen gels is done by MDA-MB-231 cells, and a recent study also shows that linear de novo formation of collagen by a coculture featuring MDA-MB-231 cells occurs. Our group recently uncovered an axis used by a trio of cells to deposit linear collagen type VI. Specifically, MDA-MB-231 cells and adipose-derived stem cells cultured in a juxtacrine manner produce high levels of CCL5. The CCL5 goes on to influence neighboring fibroblasts in a paracrine manner, which then produce linear collagen VI, revealed by decellularization of the culture after 7 days. When CCL5 is blocked, linear collagen deposition is prohibited and a randomly arranged layer of ECM is produced. Moreover, when MDA-MB-231 cells are reseeded on the linear matrix, they show high levels of proliferation and formation into road-like structures, the type of organized invasive behavior seen in enhanced metastatic abilities [7]. Velez et al. mirror these findings, but with "collagen-induced network phenotypes" using MDA-MB231 cells in collagen gels. The research showed faster migration of cells on high-density gels, formation of linear cell networks stimulated by matrix architecture, and an increase in integrin $\beta 1$ expression of migrating cells [16]. Together, these works show the feasibility of in vitro investigations on linear collagen of invasive breast cancer.

A 2016 publication features a novel in vitro arrangement where Matrigels of two different densities were interfaced. The less-dense 2-mg/ml gel contained MDA-MB-231 cells, and it was seen that these cells generated linear outgrowths which allowed invasion into the more dense 10 $\mathrm{mg} / \mathrm{ml}$ gel [2]. In 2019, a study was run on the supportive role of non-cancer cells in guiding cancer cell protrusions into controlled 3D environments in vitro. The novel models involved tumor spheroids containing cancerassociated fibroblasts (CAFs) and cancer cells in a 1.9-mg/ $\mathrm{ml}$ collagen type 1 solution. Cancer cells (pancreatic, lung adenocarcinoma) via integrin $\alpha 5 \beta 1$, bind to fibrillary fibronectin on the CAF membrane which provides a platform for migration [17]. Critically, it has been seen in breast tumor spheroids that CAFs are necessary for the secretion of ECM onto polycaprolactone scaffolds, which were later decellularized and seeded with MDA-MB-231 to create tumoroids [18]. This is yet another study using decellularization that shows the power of fibroblasts in the creation of breast cancer ECM.

\section{TACS in vivo}

The hypothetical clinical role of TACS3 was substantiated in 2011. Not only was the TACS3 linear collagen presentation detectable on $4 \mu \mathrm{m}$ histology sections across 196 human breast tumor samples, but a worse prognosis followed TACS3 biopsy presence [5]. Considering the prognostic value of this work, a machine learning model was developed to catalog and measure TACS3 in almost 200 biopsy samples and was found to be as accurate as three independent human observers as a structural biomarker in breast cancer histopathology [19].

The studies resulting from a PubMed keyword search featuring "linear collagen" AND "breast cancer," "tumor associated collagen signatures" AND "breast," "oriented collagen" AND "metastatic" pertain to aligned collagen patterns at the breast tumor boundary $[1-3,5,6,19-31]$ (Fig. 2). The upward trend of the number of these publications highlights the momentum which the field gains annually. The collective goal of these publications is currently to establish TACS as a legitimate, overlooked tumor feature and to adopt TACS as a prognostic histopathology tool.

\section{Where to go from here?}

The linearized outer coating of the invasive breast tumor, characterized by TACS3, bestows a range of prometastatic features $[2,5,32]$. There is precedent for a full investigation into the diagnostic/therapeutic impact of TACS3. While past in vitro literature focuses largely on collagen hydrogels, biasing the models to collagen type 1, the work from 2019 to 2020 shows the power of de novo collagen creation and decellularization, marking a huge step in the right direction of unbiased ECM study $[7,18]$. The research of TACS3 in vivo will be greatly boosted by further work on the proteomic composition and novel target identification of TACS3 structures. This will allow the field to step away from SHG [10] and towards specialized staining which can inform tumor progression. The finding of collagen VI at the tumor border $[7,33,34]$ is especially interesting given the nature of collagen VI breakdown products. Endotrophin (ETP) is an enzymatically generated fragment of the $\mathrm{C} 5$ residue from the $\alpha 3$ chain of collagen VI. It is automatically cleaved from the chain once the chain has left the cell body, making collagen VI both a structural and signaling protein [35]. ETP is in such high quantities in breast cancer patients that it is detectable in the blood [36] and acts as a pro-fibrotic, pro-EMT chemoattractant. Similarly, ETP has shown to increase patient resistance to cisplatin, and when blocked with antibodies in a mouse model, tumor growth is limited alongside increased 


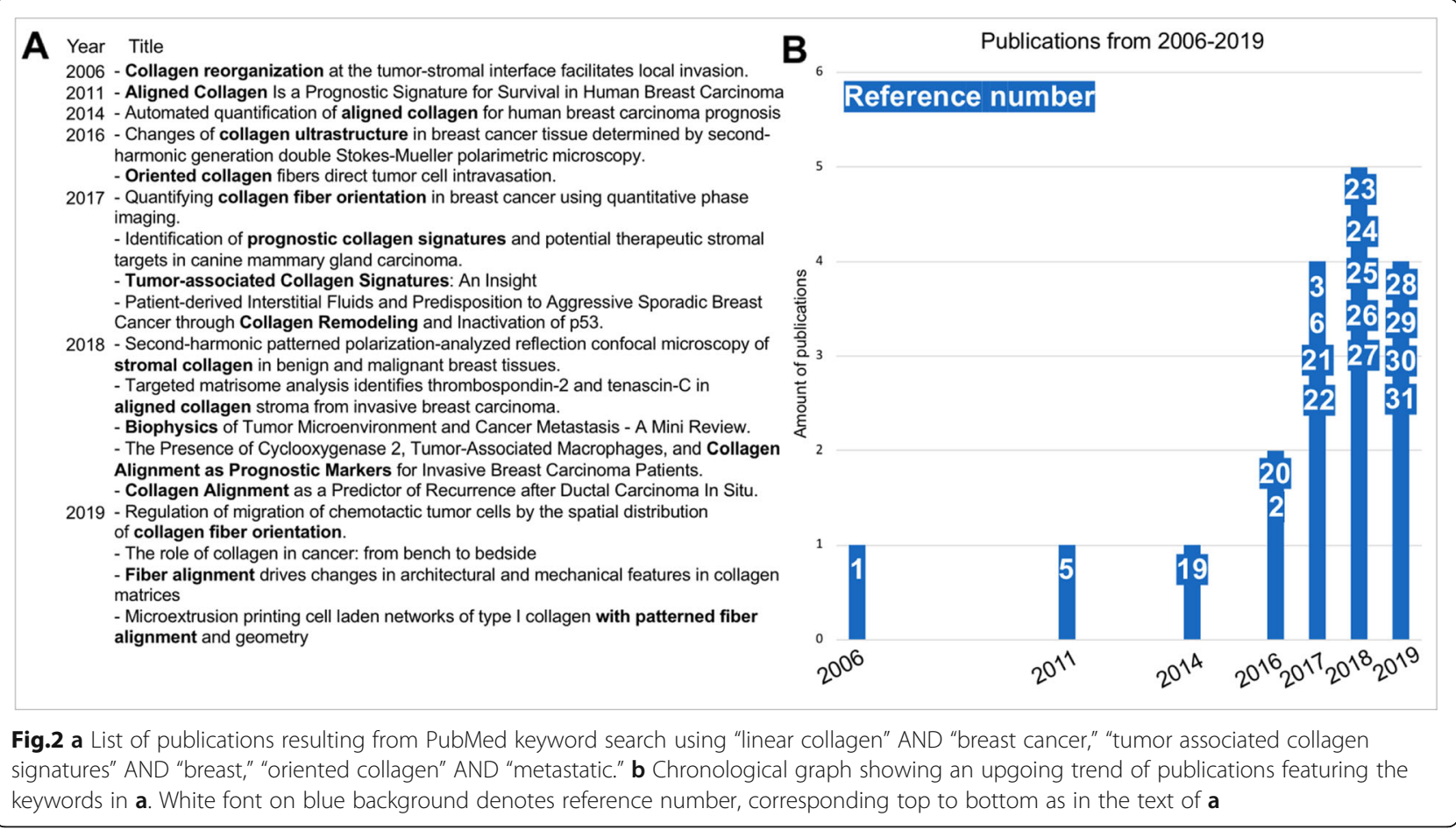

sensitivity to chemotherapy [37]. An unanswered question of breast cancer ECM research, and likely the next most important, is the biomechanical properties of the collagen. There are no studies on the stiffness of collagen or viscoelasticity of cells interacting with the matrix, which represent large knowledge gaps in the prooncogenic nature of TACS. Conceptually, it is important to note that once TACS3 structures are present at the tumor border, it is likely that the tumor has already metastasized [5]. Therefore, studies should be focused on intervention therapy, to prevent the tumor from building the structures in the first place. The role of CCL5 has been described by our group in the formation of linearized breast cancer collagen, and while there is more confirmatory work to be done, blocking CCL5 presents a logical first step towards the goal of limiting triple-negative breast tumor evolution.

\section{Conclusion}

As new research pertaining to tumor architecture emerges, it stands to inform oncologic management of the tumor, histopathological criteria, and surgical management. Existing literature across many labs reveal the in vivo TACS model to be reproducible and credible, and in vitro work to be informative and accurate. The trend of publications referenced herein highlights an increased focus on this developing model. A full understanding of TACS will further galvanize prognostics and potentially change the course of therapeutics and surgical practices. Perhaps the most impactful arm of research will be the preventative pharmacological studies, which should aim to limit TACS3 formation altogether.

\section{Acknowledgements}

The majority of the references in the "Tumor-associated collagen signatures" section originate from the Keely Lab in Madison, University of Wisconsin. Dr. Patricia Keely was the senior author on these landmark papers which coined and investigated TACS. In June 2017, she passed away from advanced pancreatic cancer at age 54 .

\section{Authors' contributions}

EB: manuscript conceptualization, literature review, writing, and figure construction. MS: manuscript editing, literature review, and writing. H-GM: study support, supervision, and manuscript editing. DD: Study support, supervision, and manuscript editing. The authors read and approved the final manuscript.

\section{Funding}

This study was supported by the Translational Medicine Program of the TUM School of Medicine and by a grant from the TUM Commission of Clinical Research to Dominik Duscher

\section{Availability of data and materials Not applicable}

Ethics approval and consent to participate

Not applicable

\section{Consent for publication}

All authors agree.

\section{Competing interests}

Not applicable

Received: 28 January 2020 Accepted: 23 June 2020

Published online: 02 July 2020

\section{References}

1. Provenzano PP, Eliceiri KW, Campbell JM, Inman DR, White JG, Keely PJ. Collagen reorganization at the tumor-stromal interface facilitates local invasion. BMC Med. 2006;4(1):38. 
2. Han W, Chen S, Yuan W, Fan Q, Tian J, Wang X, et al. Oriented collagen fibers direct tumor cell intravasation. Proc Natl Acad Sci. 2016;113(40): 11208-13.

3. Majeed H, Okoro C, Kajdacsy-Balla A, Toussaint KC Jr, Popescu G, Quantifying collagen fiber orientation in breast cancer using quantitative phase imaging. J Biomed Opt. 2017;22(4):46004.

4. Nan H, Liang L, Chen G, Liu L, Liu R, Jiao Y. Realizations of highly heterogeneous collagen networks via stochastic reconstruction for micromechanical analysis of tumor cell invasion. Phys Rev E. 2018;97(3-1): 033311.

5. Conklin MW, Eickhoff JC, Riching KM, Pehlke CA, Eliceiri KW, Provenzano PP, et al. Aligned collagen is a prognostic signature for survival in human breast carcinoma. Am J Pathol. 2011;178(3):1221-32.

6. Case A, Brisson BK, Durham AC, Rosen S, Monslow J, Buza E, et al. Identification of prognostic collagen signatures and potential therapeutic stromal targets in canine mammary gland carcinoma. PLoS One. 2017;12(7):e0180448.

7. Brett E, Sauter M, Timmins E, Azimzadeh O, Rosemann M, Merl-Pham J, et al. Oncogenic linear collagen VI of invasive breast cancer is induced by CCL5. J Clin Med. 2020;9(4):991.

8. Nadiarnykh O, LaComb RB, Brewer MA, Campagnola PJ. Alterations of the extracellular matrix in ovarian cancer studied by second harmonic generation imaging microscopy. BMC Cancer. 2010;10:94.

9. Rentchler EC, Gant KL, Drapkin R, Patankar M, Campagnola PJ. Imaging collagen alterations in STICS and high grade ovarian cancers in the fallopian tubes by second harmonic generation microscopy. Cancers (Basel). 2019;11(11):1805.

10. Campagnola PJ, Loew LM. Second-harmonic imaging microscopy for visualizing biomolecular arrays in cells, tissues and organisms. Nat Biotechnol. 2003;21(11):1356-60.

11. Chen X, Nadiarynkh O, Plotnikov S, Campagnola PJ. Second harmonic generation microscopy for quantitative analysis of collagen fibrillar structure. Nat Protoc. 2012;7(4):654-69.

12. Grubbs BA, Etter NP, Slaughter WE, Pittsford AM, Smith CR, Schmitt PD. A low-cost beam-scanning second harmonic generation microscope with application for agrochemical development and testing. Anal Chem. 2019; 91(18):11723-30

13. Majeed H, Keikhosravi A, Kandel ME, Nguyen TH, Liu Y, Kajdacsy-Balla A et al. Quantitative histopathology of stained tissues using color spatial light interference microscopy (cSLIM). Sci Rep. 2019:9(1):14679.

14. Bancelin S, Nazac A, Ibrahim BH, Dokladal P, Decenciere E, Teig B, et al. Determination of collagen fiber orientation in histological slides using Mueller microscopy and validation by second harmonic generation imaging. Opt Express. 2014;22(19):22561-74.

15. Provenzano PP, Inman DR, Eliceiri KW, Trier SM, Keely PJ. Contact guidance mediated three-dimensional cell migration is regulated by rho/ROCKdependent matrix reorganization. Biophys J. 2008:95(11):5374-84.

16. Velez DO, Tsui B, Goshia T, Chute CL, Han A, Carter H, et al. 3D collagen architecture induces a conserved migratory and transcriptional response linked to vasculogenic mimicry. Nat Commun. 2017;8(1):1651.

17. Miyazaki K, Oyanagi J, Hoshino D, Togo S, Kumagai H, Miyagi Y. Cancer cell migration on elongate protrusions of fibroblasts in collagen matrix. Sci Rep. 2019:9(1):292.

18. Nayak B, Balachander GM, Manjunath S, Rangarajan A, Chatterjee K. Tissue mimetic 3D scaffold for breast tumor-derived organoid culture toward personalized chemotherapy. Colloids Surf B Biointerfaces. 2019;180:334-43.

19. Bredfeldt JS, Liu Y, Conklin MW, Keely PJ, Mackie TR, Eliceiri KW. Automated quantification of aligned collagen for human breast carcinoma prognosis. J Pathol Inform. 2014;5(1):28.

20. Golaraei A, Kontenis L, Cisek R, Tokarz D, Done SJ, Wilson BC, et al. Changes of collagen ultrastructure in breast cancer tissue determined by secondharmonic generation double stokes-Mueller polarimetric microscopy. Biomed Opt Express. 2016;7(10):4054-68.

21. Pavithra V, Sowmya SV, Rao R, Patil S, Augustine D, Haragannavar V, et al. Tumor-associated collagen signatures: an insight. World Journal of Dentistry. 2017:8:224-30

22. Kenny TC, Schmidt H, Adelson K, Hoshida Y, Koh AP, Shah N, et al. Patientderived interstitial fluids and predisposition to aggressive sporadic breast cancer through collagen remodeling and inactivation of p53. Clin Cancer Res. 2017;23(18):5446-59.

23. Okoro C, Kelkar V, Sivaguru M, Emmadi R, Toussaint KC. Second-harmonic patterned polarization-analyzed reflection confocal microscopy of stromal collagen in benign and malignant breast tissues. Sci Rep. 2018;8(1):16243.
24. Tomko LA, Hill RC, Barrett A, Szulczewski JM, Conklin MW, Eliceiri KW, et al. Targeted matrisome analysis identifies thrombospondin-2 and tenascin-C in aligned collagen stroma from invasive breast carcinoma. Sci Rep. 2018;8(1):12941.

25. Emon B, Bauer J, Jain Y, Jung B, Saif T. Biophysics of tumor microenvironment and cancer metastasis - a mini review. Comput Struct Biotechnol J. 2018;16:279-87.

26. Esbona K, Yi Y, Saha S, Yu M, Van Doorn RR, Conklin MW, et al. The presence of cyclooxygenase 2, tumor-associated macrophages, and collagen alignment as prognostic markers for invasive breast carcinoma patients. Am J Pathol. 2018;188(3):559-73.

27. Conklin MW, Gangnon RE, Spraque BL, Van Gemert L, Hampton JM, Eliceiri $\mathrm{KW}$, et al. Collagen alignment as a predictor of recurrence after ductal carcinoma in situ. Cancer Epidemiol Biomark Prev. 2018;27(2):138-45.

28. Azimzade Y, Saberi AA, Sahimi M. Regulation of migration of chemotactic tumor cells by the spatial distribution of collagen fiber orientation. Phys Rev E. 2019;99(6-1):062414

29. Xu S, Xu H, Wang W, Li S, Li H, Li T, et al. The role of collagen in cancer: from bench to bedside. J Transl Med. 2019;17(1):309.

30. Taufalele PV, VanderBurgh JA, Munoz A, Zanotelli MR, Reinhart-King CA. Fiber alignment drives changes in architectural and mechanical features in collagen matrices. PLoS One. 2019;14(5):e0216537.

31. Nerger BA, Brun P-T, Nelson CM. Microextrusion printing cell-laden networks of type I collagen with patterned anisotropy and geometry. bioRxiv. 2019:509265.

32. Hanahan D, Weinberg RA. Hallmarks of cancer: the next generation. Cell. 2011;144(5):646-74.

33. Iyengar $P$, Espina V, Williams TW, Lin Y, Berry D, Jelicks LA, et al. Adipocytederived collagen VI affects early mammary tumor progression in vivo, demonstrating a critical interaction in the tumor/stroma microenvironment. J Clin Invest. 2005;115(5):1163-76.

34. Karousou E, D'Angelo ML, Kouvidi K, Vigetti D, Viola M, Nikitovic D, et al. Collagen VI and hyaluronan: the common role in breast cancer. Biomed Res Int. 2014;2014:606458.

35. Cescon M, Gattazzo F, Chen P, Bonaldo P. Collagen VI at a glance. J Cell Sci. 2015;128(19):3525-31.

36. Willumsen N, Bager C, Karsdal MA. Matrix metalloprotease generated fragments of type VI collagen have serum biomarker potential in cancer - a proof of concept study. Transl Oncol. 2019:12(5):693-8.

37. Bu D, Crewe C, Kusminski CM, Gordillo R, Ghaben AL, Kim M, et al. Human endotrophin as a driver of malignant tumor growth. JCI Insight. 2019;5.

\section{Publisher's Note}

Springer Nature remains neutral with regard to jurisdictional claims in published maps and institutional affiliations.

Ready to submit your research? Choose BMC and benefit from:

- fast, convenient online submission

- thorough peer review by experienced researchers in your field

- rapid publication on acceptance

- support for research data, including large and complex data types

- gold Open Access which fosters wider collaboration and increased citations

- maximum visibility for your research: over $100 \mathrm{M}$ website views per year

At $\mathrm{BMC}$, research is always in progress.

Learn more biomedcentral.com/submission 